Mon. Not. R. Astron. Soc. 000, ??-?? (2020) Printed 11 May $2021 \quad$ (MN LATEX style file v2.2)

\title{
Systematic uncertainties in models of the cosmic dawn
}

\author{
Jordan Mirocha, ${ }^{\star \dagger}$ Henri Lamarre, Adrian Liu ${ }^{\ddagger}$ \\ Department of Physics \& McGill Space Institute, McGill University, 3600 Rue University, Montréal, QC, H3A 2T8
}

11 May 2021

\begin{abstract}
Models of the reionization and reheating of the intergalactic medium (IGM) at redshifts $z \gtrsim 6$ continue to grow more sophisticated in anticipation of near-future $21-\mathrm{cm}$, cosmic microwave background, and galaxy survey measurements. However, there are many potential sources of systematic uncertainty in models that could bias and/or degrade upcoming constraints if left unaccounted for. In this work, we examine three commonly-ignored sources of uncertainty in models for the mean reionization and thermal histories of the IGM: the underlying cosmology, halo mass function (HMF), and choice of stellar population synthesis (SPS) model. We find that cosmological uncertainties affect the Thomson scattering optical depth at the few percent level and the amplitude of the global 21-cm signal at the $\sim 5-10 \mathrm{mK}$ level. The differences brought about by choice of HMF and SPS models are more dramatic, comparable to the $1 \sigma$ error-bar on $\tau_{e}$ and a $\sim 20 \mathrm{mK}$ effect on the global $21-\mathrm{cm}$ signal amplitude. Finally, we jointly fit galaxy luminosity functions and global 21-cm signals for all HMF /SPS combinations and find that (i) doing so requires additional free parameters to compensate for modeling systematics and (ii) the spread in constraints on parameters of interest for different HMF and SPS choices, assuming $5 \mathrm{mK}$ noise in the global signal, is comparable to those obtained when adopting the "true" HMF and SPS with $\gtrsim 20$ $\mathrm{mK}$ errors. Our work highlights the need for dedicated efforts to reduce modeling uncertainties in order to enable precision inference with future datasets.
\end{abstract}

Key words: galaxies: high-redshift - intergalactic medium - galaxies: luminosity function, mass function - dark ages, reionization, first stars - diffuse radiation.

\section{INTRODUCTION}

Many ongoing and near-future experiments are designed in large-part to provide astrophysical and cosmological constraints on the "cosmic dawn," when the first stars and galaxies formed. For example, galaxy surveys searching for high redshift $z \gtrsim 6$ galaxies will continue piecing together the luminosity function of galaxies over cosmic time (Williams et al., 2018; Behroozi et al., 2020), while cosmic microwave background (CMB) measurements constrain the rough timing and duration of reionization from the Thomson scattering optical depth, $\tau_{e}$, and kinetic Sunyaev-Z'eldovich effect (Planck Collaboration et al., 2018; Reichardt et al., 2020). 21-cm experiments target neutral hydrogen itself, and are thus direct probes of the mean ionization and thermal histories, as well as their topology (Furlanetto, 2006; Pritchard \& Loeb, 2012).

Of course, the native form of measurements provided

\footnotetext{
^ jordan.mirocha@mcgill.ca

$\dagger$ CITA National Fellow

‡acliu@physics.mcgill.ca
}

by the aforementioned probes, e.g., luminosity functions, $\tau_{e}$, and 21-cm spectra, are not straightforward to interpret. As a result, forward models of galaxies and reionization are used to predict these quantities, and, once coupled to sampling algorithms like Markov Chain Monte Carlo (MCMC), can be used to infer the underlying parameters of the model from measurements. For example, UV luminosity functions (UVLFs) of high- $z$ galaxies can constrain the star formation efficiency (SFE; $f_{*}$ ) of galaxies (Mason et al., 2015; Sun \& Furlanetto, 2016), while the reionization and thermal histories are sensitive to, e.g., the product of $f_{*}$, the escape fraction of ionizing photons $f_{\text {esc }}$, and assumptions about galaxies beyond detection limits (Robertson et al., 2015; Bouwens et al., 2015a). Meanwhile, 21-cm probes are sensitive also to the minimum mass of halos capable of forming stars, $M_{\min }$, and the efficiency of X-ray and non-ionizing UV photon production in high- $z$ galaxies (Barkana \& Loeb, 2005; Furlanetto, 2006; Pritchard \& Furlanetto, 2007; Mesinger et al., 2013). Additionally, 21-cm experiments can in principle be used to constrain fundamental physics, given that warm and interacting dark matter models affect the formation of structure on small scales (Sitwell et al., 2014; Lopez-Honorez 
Mirocha, Lamarre, ES Liu

et al., 2016; Schneider, 2018; Muñoz et al., 2020) and modulate the IGM thermal history relative to the predictions of $\Lambda$ cold dark matter (CDM) models (Tashiro et al., 2014; Barkana, 2018; Muñoz \& Loeb, 2018; Boddy et al., 2018; Fialkov et al., 2018). Indeed, many experiments are underway, and have provided interesting limits on both the reionization and thermal histories (e.g., Bowman et al., 2018; Monsalve et al., 2017; Singh et al., 2018).

Several parameter inference pipelines have emerged in recent years to interpret the impending deluge of constraints on the cosmic dawn. Semi-empirical or semi-analytic models of galaxy formation are now commonly employed to connect constraints on UVLFs, the mean reionization history, and ionizing background after reionization (Finkelstein et al., 2019; Tacchella et al., 2018; Behroozi et al., 2019; Yung et al., 2019), as well as global 21-cm signals (Mirocha et al., 2017). Semi-numerical models of reionization have emerged to efficiently model the 21-cm background in 3-D (Mesinger \& Furlanetto, 2007; Mesinger et al., 2011; Santos et al., 2010; Fialkov et al., 2013; Hutter et al., 2020), and have recently been coupled to MCMC samplers (Greig \& Mesinger, 2015, 2017) and emulators (Kern et al., 2017; Cohen et al., 2020) to enable joint galaxy survey-21-cm inference (Park et al., 2019; Qin et al., 2020).

Recent forecasts largely find that precision constraints on parameters of interest are well within the reach of current facilities. For example, 21-cm measurements can constrain the population-averaged values of $f_{*}, f_{\text {esc }}$, above $M_{\min }$ (Mirocha et al., 2015; Greig \& Mesinger, 2017), warm dark matter (Muñoz et al., 2020), and even cosmological parameters (Liu et al., 2016; Kern et al., 2017). If limited only by thermal noise, current experiments are expected to reach the cosmic variance floor in relatively short order (Muñoz \& Cyr-Racine, 2020). Of course, such forecasts are based on ideal instruments, foregrounds, and noise-luxuries yet to be achieved in real experiments, for which systematic uncertainties are the main impediment to progress (see, e.g., Liu \& Shaw 2020 for a description of some of the main issues).

Systematic uncertainties are not limited to the realm of experiment. Theoretical models are also uncertain, in part due to numerical approximations made to accelerate computations, but also because of assumptions made in the modeling. Some of these assumptions are not necessarily parameterizable, and thus may be difficult to marginalize over when computing parameter constraints. For example, the semi-numerical approach to reionization is an approximation employed to avoid running expensive radiative transfer simulations, and appears to work at the level of $\sim 10-20 \%$ (Zahn et al., 2011; Majumdar et al., 2014; Hutter, 2018). It is not clear that the level of uncertainty is invariant with respect to model parameters or implementations, and so it is not clear how to generically inflate uncertainties on model parameters of interest. Other aspects of cosmic dawn modeling may be even more difficult to pin down as they are unrelated to numerical approximations, e.g., choosing the "right" stellar population synthesis model or halo mass function.

There is no doubt that observational issues are still the most pressing. However, given that the sensitivity of the current generation of 21-cm experiments is sufficient for detection of the global 21-cm signal and power spectrum, a breakthrough in the reduction of systematics could lead to rapid transition from crude upper limits to proper constraints on astrophysical and cosmological parameters. As a result, it is fruitful to consider the limitations of current models, so that particularly weak areas of models can be identified and targeted for priority work. We also hope that doing so may also inspire the community to critically examine the degree of precision needed for different science questions.

While there are many potential sources of systematic uncertainty in models, in this work we focus on three main effects associated with seemingly innocuous modeling decisions: (i) the neglect of uncertainties in cosmological parameters, (ii) the halo mass function (HMF), and (iii) stellar population synthesis (SPS) codes. While cosmological parameter variations can in principle be included self-consistently and marginalized over, they rarely are, especially in semi-numeric codes for which re-generating the cosmology-dependent density and velocity fields can be the most expensive part of the model. Unlike the cosmological parameters, the choice of HMF and SPS model does not lie along a continuum of possibilities. In other words, these "parameters" are switches rather than knobs. Other likely causes of uncertainty include numerical techniques (e.g., two-zone vs. semi-numeric vs. fully-numerical), or choice of parameterization for galaxy properties (e.g., mass-dependent vs. mass-independent parameters, inclusion of multiple sources populations). These areas are perhaps equally deserving of attention, though we defer them to future work.

The structure of the paper is as follows. In $\S 2$ we review our modeling approach, giving special attention to the components most likely to be uncertain. We present our main results in $\S 3$ and conclude in $\S 4$.

\section{METHODS}

Our approach to modeling the reionization and re-heating of the IGM closely follows early two-zone models for the IGM (Furlanetto, 2006; Pritchard \& Loeb, 2010) though we use updated models for the X-ray background and galaxies following Mirocha (2014) and Mirocha et al. (2017), respectively. We provide a brief summary of the model below, drawing attention to the components sucseptible to the modeling uncertainties we investigate. Our results can be re-created using the publicly-available ARES code ${ }^{1}$.

To begin, we assume that galaxies inhabit dark matter halos in a 1:1 fashion and that star formation is fueld by the inflow of pristine gas from the IGM. This means that the star formation rate $\dot{M}_{*}$ (SFR) in galaxies is directly related to the mass accretion rate $\dot{M}_{h}$ (MAR) of dark matter halos,

$$
\dot{M}_{*}=f_{*} f_{b} \dot{M}_{h}
$$

were $f_{*}$ is the star formation efficiency (SFE) and $f_{b}$ is the cosmic baryon fraction. We assume that the SFE as a double-power law (DPL) in halo mass,

$$
f_{*}\left(M_{h}\right)=\frac{f_{*, 10} \mathcal{C}_{10}}{\left(\frac{M_{h}}{M_{\mathrm{p}}}\right)^{-\alpha_{\mathrm{lo}}}+\left(\frac{M_{h}}{M_{\mathrm{p}}}\right)^{-\alpha_{\mathrm{hi}}}}
$$

where $f_{*, 10}$ is the SFE at $10^{10} M_{\odot}, M_{p}$ is the mass at which

1 https://ares.readthedocs.io/en/latest/ 
$f_{*}$ peaks, and $\alpha_{\text {hi }}$ and $\alpha_{\text {lo }}$ describe the power-law index at masses above and below the peak, respectively. The additional constant $\mathcal{C}_{10} \equiv\left(10^{10} / M_{p}\right)^{-\alpha_{\mathrm{lo}}}+\left(10^{10} / M_{p}\right)^{-\alpha_{\mathrm{hi}}}$ is introduced to re-normalize the standard DPL formula to $10^{10} M_{\odot}$, rather than the peak mass. We model the MAR following Furlanetto et al. (2017), and assume halos grow at fixed number density, in which case $\dot{M}_{h}$ can be inferred from the evolution in the HMF. Though idealized, it has the advantage of preserving self-consistency, and does appear to agree well with the results of N-body simulations (Trac et al., 2015), at least for relatively massive halos at $z \lesssim 10$. The MAR is yet another potential source of modeling uncertainty that may be comparable to the effects we explore here (Schneider et al., 2020), though we defer investigation of this possibility to future work.

In this work, we only consider the rest-ultrioviolet continuum emission of galaxies $(1600 \AA)$, which is probed by recent observations with Hubble (Bouwens et al., 2015b; Finkelstein et al., 2015) targeting galaxies at $z \gtrsim 4$. Because the rest-UV continuum probes young stars, it is a good tracer of recent star formation and thus the SFR of galaxies. Under the assumption of a constant SFR, the UV luminosity of a galaxy will asymptote to a constant value on timescales of order $\sim 100 \mathrm{Myr}$. In this limit, the UV luminosity can be modeled simply as

$$
L_{\mathrm{UV}}=l_{\mathrm{UV}} \dot{M}_{*}
$$

where $l_{\mathrm{UV}}$ carries units of $\mathrm{erg} \mathrm{s}^{-1} \mathrm{~Hz}^{-1}\left(M_{\odot} \mathrm{yr}^{-1}\right)^{-1}$ and is often written instead via its reciprocal $\kappa_{\mathrm{UV}} \equiv l_{\mathrm{UV}}^{-1}$.

Finally, with these assumptions, the UV luminosity function is simply

$$
d \phi\left(L_{\mathrm{UV}}\right)=\frac{d n_{h}}{d M_{h}} \frac{d M_{h}}{d L_{\mathrm{UV}}} d L_{\mathrm{UV}}
$$

Here, $d n_{h} / d M_{h}$ is the halo mass function, which we compute using the HMF code (Murray et al., 2013a), which relies on CAMB for the matter power spectrum (Lewis et al., 2000). For an assumed value of $l_{\mathrm{UV}}$, one can calibrate $f_{*}$ empirically by fitting UVLF constraints from any number of studies (Bouwens et al., 2015b; Finkelstein et al., 2015) using Eqs. (1)-(3) to compute $d M_{h} / d L_{\mathrm{UV}}$. We note that several aspects of this model have since been generalized, e.g., selfconsistent dust reddening, "bursty star" formation histories (Mirocha et al., 2020; Mirocha, 2020), though we neglect these complications for the duration of this paper, as they will not obviously exacerbate (or mitigate) the sources of uncertainty we explore here. For results presented in the remainder of this paper, we use models that neglect dust reddening (Fig. 3-6; mostly for illustrative purposes), as well as models that employ a relatively standard dust correction using the Meurer et al. (1999) IRX- $\beta$ relation and $M_{\mathrm{UV}}-$ $\beta$ relations from Bouwens et al. (2014) (Fig. 7-8). In each case, fits to the relevant datasets are performed using EMCEE (Foreman-Mackey et al., 2013).

With a model for sources in hand, we can proceed to modeling the mean reionization history and global $21-\mathrm{cm}$ signal. The differential brightness temperature $\delta T_{b}$ is given by

$\delta T_{b} \simeq 27\left(1-x_{\mathrm{H}}{ }_{\text {II }}\right)\left(\frac{\Omega_{\mathrm{b}, 0} h^{2}}{0.023}\right)\left(\frac{0.15}{\Omega_{\mathrm{m}, 0} h^{2}} \frac{1+z}{10}\right)^{1 / 2}\left(1-\frac{T_{\gamma}}{T_{\mathrm{S}}}\right)$, where $x_{\mathrm{H} \text { II }}=Q_{\mathrm{HII}}+\left(1-Q_{\mathrm{HII}}\right) x_{e}$ is the volume-averaged ionized fraction, and

$$
T_{\mathrm{S}}^{-1} \approx \frac{T_{\gamma}^{-1}+x_{c} T_{\mathrm{K}}^{-1}+x_{\alpha} T_{\alpha}^{-1}}{1+x_{c}+x_{\alpha}} .
$$

is the spin temperature. The spin temperature quantifies the level populations in the hyperfine singlet and triplet states, and is set by a combination of collisional coupling (quantified by $x_{c}$; Zygelman, 2005), radiative coupling through the Wouthuysen-Field effect (quantified by $x_{\alpha}$; Wouthuysen, 1952; Field, 1958) and microwave background temperature, $T_{\gamma}$. Equation (5) assumes the cosmic mean density and neglects relative velocities along the line of sight, as is appropriate for the global 21-cm signal.

Our model partitions the IGM into two distinct phases: an ionized phase, characterized by its volume-filling factor $Q_{\text {HII }}$, and a "bulk IGM" phase, characterized by its temperature and $\mathrm{Ly}-\alpha$ intensity. To evolve the gas temperature in the bulk IGM phase, we solve the uniform background problem as outlined in Mirocha (2014), essentially a high- $z$ implementation of the Haardt \& Madau (1996) algorithm. Assuming a multi-colour disk spectrum for X-ray emission (Mitsuda et al., 1984) with no host-galaxy absorption, we solve for the mean intensity of the cosmic X-ray background as a function of redshift and photon energy, $J_{\nu}(z)$, and integrate over $J_{\nu}$ (weighted by bound-free absorption crosssections) to obtain the photo-heating and ionization rates as a function of redshift. We assume a fully-neutral IGM, in which case the bound-free optical depth of the IGM to X-ray photons can be tabulated ahead of time for an efficiency boost. The intensity of the Ly- $\alpha$ background, $J_{\alpha}$, which sets the strength of Wouthuysen-Field coupling, is computed in an analogous fashion. Note that this part of the model is cosmology-dependent, as $J_{\nu}$ depends on the Hubble parameter - we self-consistently include the cosmology dependence in this computation. See $§ 2.2-2.3$ in Mirocha (2014) for more details.

Because the mean-free path of ultraviolet photons is short, we relate the growth rate of the fractional ionized volume, $Q_{\mathrm{HII}}$, directly to the ionizing photon production rate, i.e.,

$$
\frac{d Q_{\mathrm{HII}}}{d t}=\frac{f_{\mathrm{esc}} N_{\mathrm{ion}} \dot{\rho}_{*}}{n_{\mathrm{H}}}-\alpha_{\mathrm{HII}} C n_{e} Q_{\mathrm{HII}}
$$

where $f_{\text {esc }}$ is the escape fraction of ionizing photons, $N_{\text {ion }}$ is the number of ionizing photons emitted per unit stellar mass, and $\dot{\rho}_{*} \equiv \int d M_{h} \dot{M}_{*} d n_{h} / d M_{h}$ is the cosmic star formation rate density. The recombination rate is the product of the clumping factor $C$, which we set to unity for simplicity, the electron number density $n_{e}$, and the case A recombination coefficient, $\alpha_{\mathrm{HII}}$.

\subsection{Sources of Modelling Uncertainty}

Having established our basic underlying model of UVLFs and the global $21 \mathrm{~cm}$ signal, we now highlight several potential sources of theoretical uncertainty.

Latent in virtually every expression in this section are assumptions about cosmological parameters. The 21-cm background itself depends directly on $\Omega_{b, 0}$ and the Hubble parameter, as it involves an integral along the line of sight, 
but also indirectly, as the opacity of the IGM to X-rays similarly depends on $\Omega_{b, 0}$, the primordial helium abundance, and the Hubble parameter.

The HMF, while possible to compute analytically under idealized circumstances, is likely more complicated in reality. Recent numerical simulations (e.g., Tinker et al., 2010) suggest that the HMF lies somewhere between the classic analytic models (Press \& Schechter, 1974; Sheth et al., 2001) often used in reionization models. The results of N-body simulations can be reproduced via fitting functions, though these vary from model to model due to differences in gravity solver and/or halo finder used in the analysis (Knebe et al., 2013a,b), and themselves may be insufficient for precision work (Bhattacharya et al., 2011). Most numerical simulations run to date are not optimized for high- $z$ (though see, e.g., Reed et al., 2007), nor has there been a systematic exploration of the agreement between different fitting functions at $z \gtrsim 2$. The fact that variations from one HMF fitting function to the next are larger than those caused by cosmological parameter variations for a single HMF at low- $z$ (Murray et al., 2013b) suggests this is a problem worth investigating at high- $z$ as well (see, e.g., Lopez-Honorez et al., 2016). In this work, we investigate the Press \& Schechter (1974), Sheth et al. (2001), and the Tinker et al. (2010) form of the mass function, the latter of which is a representative example that lies between. Other recent results are comparable to Tinker et al. (2010) (Warren et al., 2006; Reed et al., 2007; Bhattacharya et al., 2011), at least at low redshift where comparisons are most common (Murray et al., 2013b).

Next, the conversion factor from SFR to luminosity, $\kappa_{\mathrm{UV}}$ (see Eq. 3), relies on assumptions about stellar evolution, atmospheres, and initial mass function (IMF). Like the HMF, pieces of $\kappa_{\mathrm{UV}}$ are in principle "knobs" just like the cosmological parameters, e.g., the stellar initial mass function (IMF), which is generally taken to be a power-law or broken power-law. However, in practice, it is common to choose an SPS code and implicitly adopt the IMF used within it. In this work we use the BPASs version 1.0 models $^{2}$ (Eldridge \& Stanway, 2009) as well as the original STARBURST99 models (Leitherer et al., 1999).

In Figure 1 we show the HMFs adopted in this work. We show both the cumulative HMF (top) and fraction of matter in collapsed halos, $f_{\text {coll }}$, above the atomic cooling threshold $\left[M_{h}\left(T_{\text {vir }}=10^{4} \mathrm{~K}\right) \equiv m_{4}\right.$; bottom $]$. At $z=6$, the disagreement between models is most clear in massive halos, $M_{h} \gtrsim 10^{11} M_{\odot}$, though upon close inspection differences are present in low-mass halos, and the sense of the disparity between fitting functions reverses. At $z \gtrsim 10$, the disagreement is noticeable at all masses. The bottom panel is perhaps the clearer metric for reionization models, where the production rate of UV and X-ray photons is often linked directly to the rate at which mass collapses into halos. At $z \sim 20$, the spread is nearly an order of magnitude, while at $z \sim 6$ it is still a factor of $\sim 2$. In all cases, variations in

2 We find that the BPAss version 2.1 models (Eldridge et al., 2017) differ from the version 1.0 models by $\lesssim 20 \%$ in the production of UV photons relevant for UVLFs, reionization, and Lyman- $\alpha$ coupling of the 21 -cm line for stellar ages $\lesssim 100$ Myr.
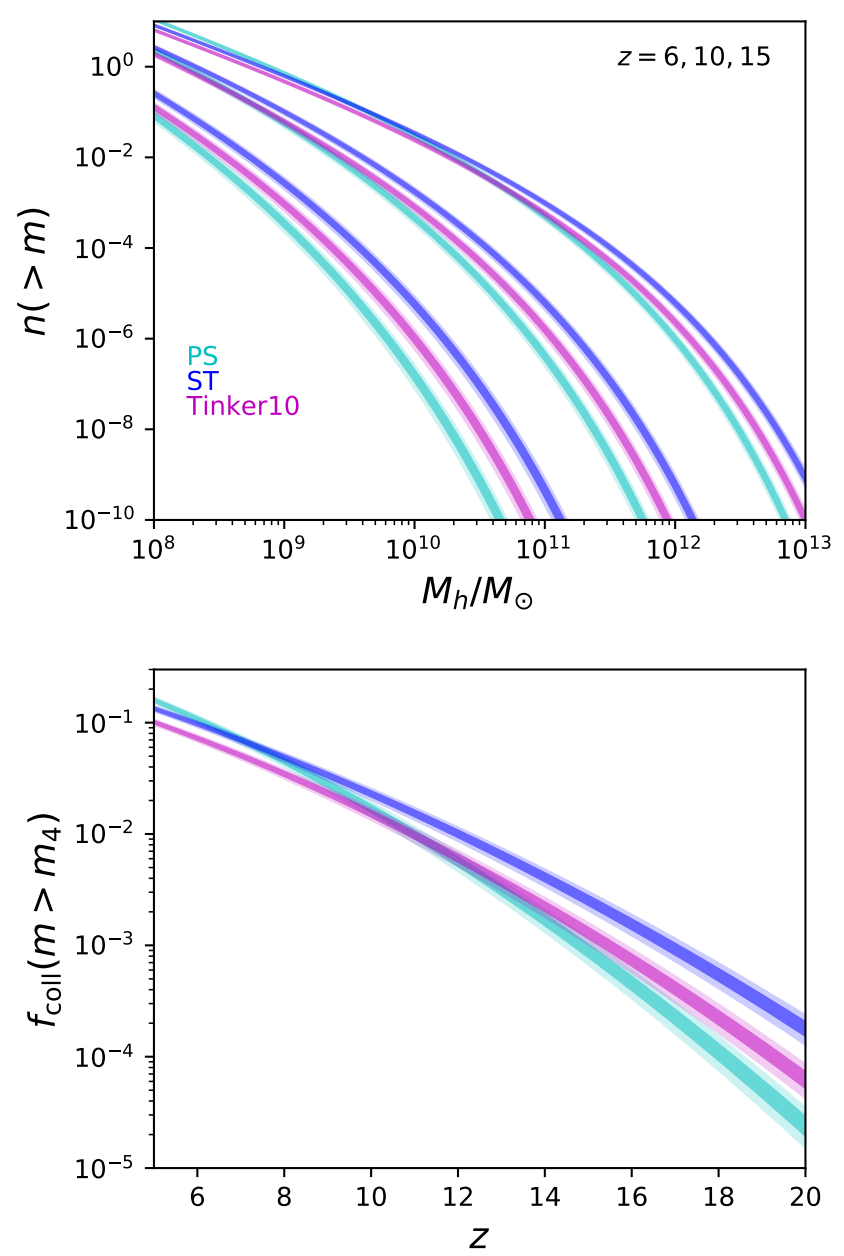

Figure 1. Difference in cumulative HMF (top) and collapse fraction (bottom) for different fitting functions. Width of each line is due to variations in cosmological parameters ( 1 and $2 \sigma$ contours drawn for each), while each colour indicates a different fitting function: Press \& Schechter (1974) (PS; cyan), Sheth et al. (2001) (ST; blue), and Tinker et al. (2010) (Tinker10; magenta). In the top panel, the three sets of curves indicate redshifts $z=6,10$, and 15 from top to bottom.

the cosmological parameters (shaded regions) has a smaller effect than the choice of fitting function (different colours).

In Figure 2, we compare the SPS models used in this work. First, in the top panel, we show an example spectral energy distribution (SED) for a constant SFR and metallicity of $\mathcal{Z}=0.004$ using both BPASs (black) and STARBURST99 (blue). There is a clear offset in normalization between the two codes, with STARBURST99 producing more overall emission (per $M_{\odot} \mathrm{yr}^{-1}$ ) at all wavelengths. To investigate the $\lambda$-dependent nature of the offset, we scale the STARBURST99 spectrum so that it matches BPASS at $1600 \AA$ (blue dashed). It is now clear that, in addition to producing a stronger nonionizing continuum, STARBURST99 also has a higher ratio of non-ionizing to ionizing emission than BPASS.

The SED effects are explored further in the bottom panel of Fig. 2. For a series of metallicities, we compute the ratio of ionizing (black) and Lyman-Werner band (blue) luminosities to the $1600 \AA$ luminosity for each code, and then 

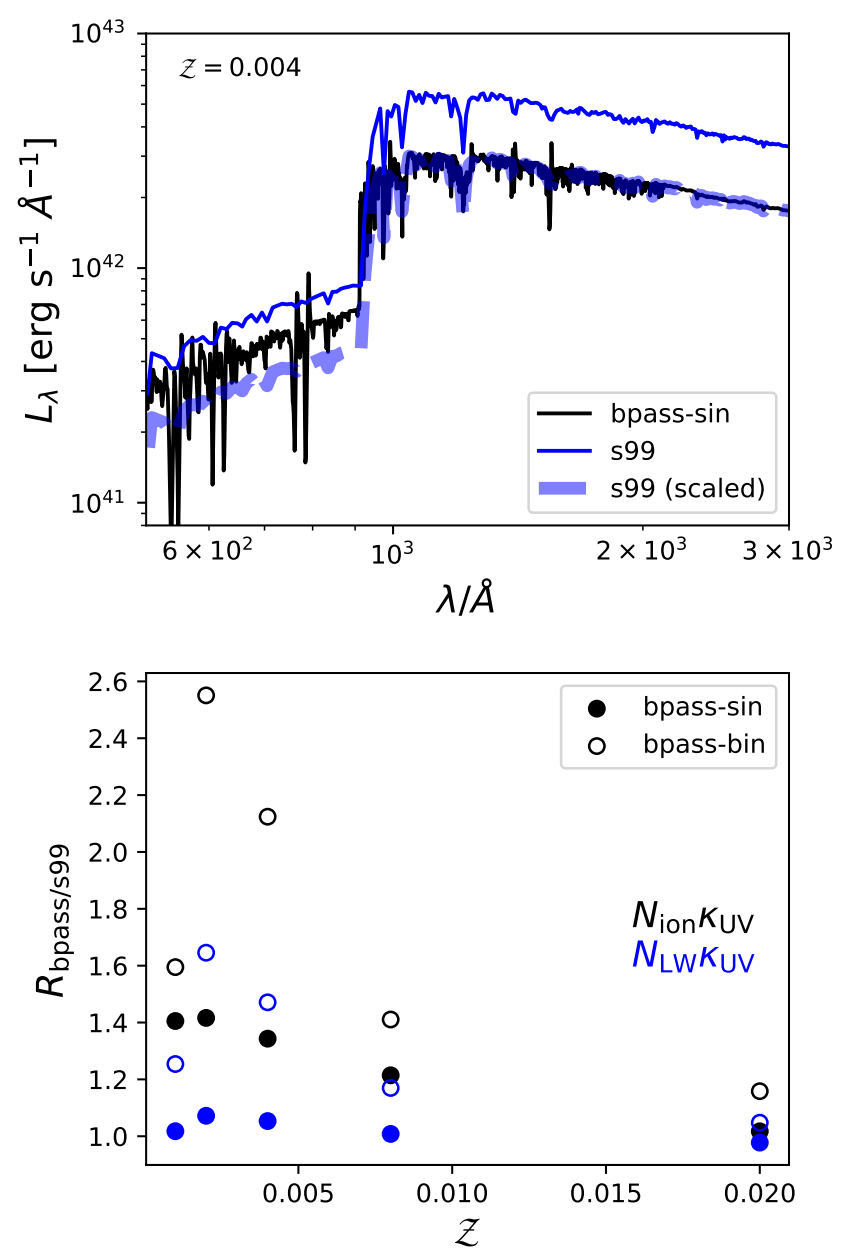

Figure 2. Difference in rest-UV SEDs of galaxies as computed with BPAss (black) vs. STARBURsT99. Top panel shows the SED of a metallicity $\mathcal{Z}=0.004$ stellar population forming stars continuously for the last $100 \mathrm{Myr}$, while the bottom panel compares the ratio of ionizing $\left(N_{\text {ion }}\right)$ and Lyman-Werner $\left(N_{\mathrm{LW}}\right)$ luminosities to the $1600 \AA$ continuum for each code at a series of stellar metallicities.

plot the ratio of values for each code, i.e.,

$$
R \equiv \frac{\left(N \times \kappa_{\mathrm{UV}}\right)_{\mathrm{bpass}}}{\left(N \times \kappa_{\mathrm{UV}}\right)_{\mathrm{s} 99}}
$$

where $N$ is either $N_{\text {ion }}$, the number of ionizing photons emitted per stellar baryon, or $N_{\mathrm{LW}}$, the equivalent quantity in the Lyman-Werner band.

The rationale here is that the rest-ultraviolet $\sim 1600 \AA$ emission is the only part of the spectrum that is (generally) observable - at fixed $\kappa_{\mathrm{UV}}$, UVLFs constrain $f_{*}$, so any differences in mean reionization history and global $21-\mathrm{cm}$ signal will largely be determined by the product $N_{\text {ion }} \kappa_{\mathrm{UV}}$ and $N_{\mathrm{LW}} \kappa_{\mathrm{UV}}$, respectively. Indeed, such differences can be many tens of percent, perhaps a factor of $\sim 2$ in extreme cases, particularly at low metallicity $Z \lesssim 0.01$ and when adopting the BPASs binaries models ("bpass-bin"; open symbols). We focus only on single star models throughout this work in order to make a more fair comparison between BPASS and STARBURST 99 .

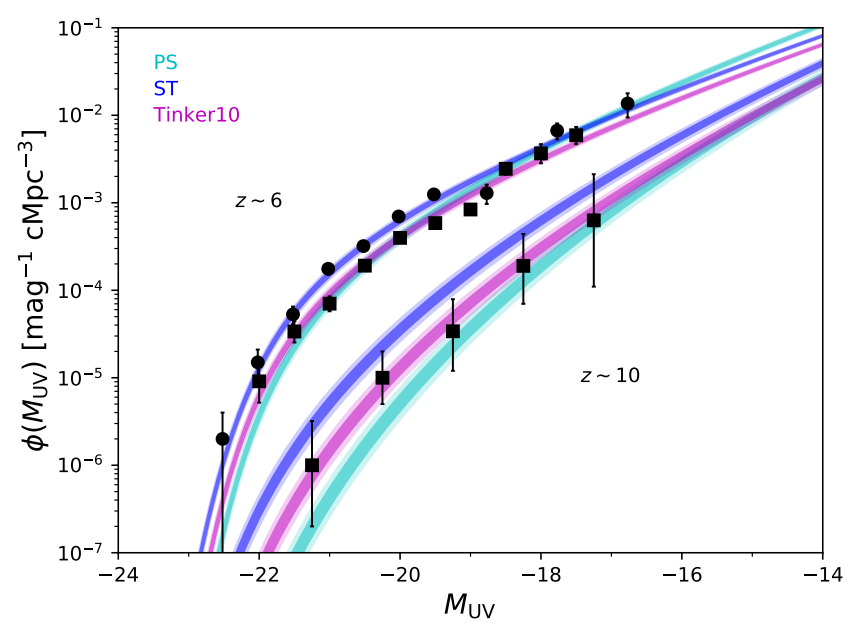

Figure 3. Difference in UVLFs caused by cosmology and HMF variations. Linestyle and colour conventions are the same as Figures 4 and 6 . Data at $z \sim 6$ are from Bouwens et al. (2015b) (circles) and Finkelstein et al. (2015) (squares), while $z \sim 10$ measurements are from Oesch et al. (2018). Note that there are three bands shown for each redshift.

\section{RESULTS}

We now present our main results, consisting of models run with $10^{3}$ elements from the Planck chain (planck_TTTEEE_lowl_lowE), with three different HMFs, using both STARBURST99 and BPASS SPS models. For each cosmological model, we re-generate (i) the recombination history using Cosmorec (Chluba \& Thomas, 2011), (ii) the optical depth of the IGM used to compute the X-ray background, and (iii) the HMF using HMF (Murray et al., 2013a). The cosmological model number is subsequently used as a free parameter, which indicates the appropriate lookup table to use for each of these three quantities, as well as the cosmological parameter values used elsewhere in the model.

We begin with a pure forward modeling approach to examine the extent to which these different assumptions affect predictions for the mean reionization history, global $21-\mathrm{cm}$ signal, and UVLFs. For this exercise, we adopt the Mirocha et al. (2017) model calibration, which fit the $z \simeq 6$ Bouwens et al. (2015b) UVLFs with the model described in $\S 2$ under the assumption of no dust reddening (for illustrative purposes). These results are shown in Figures 3-6, in which the four parameters governing $f_{*}$ (see Eq. 2) are held fixed to their best-fit values: $f_{*, 10}=0.05, M_{\mathrm{p}}=2.8 \times 10^{11} M_{\odot}$, $\alpha_{\mathrm{lo}}=0.49$, and $\alpha_{\mathrm{hi}}=-0.61$. Then, we will turn our attention to the inference problem, and quantify the degree to which parameter constraints are worsened when including these "new" sources of uncertainty, while holding roughly fixed the UVLFs and global 21 -cm signal via joint fitting. In this case, we employ the standard IRX- $\beta$-based dust correction to more closely mimic the procedure likely to be applied on upcoming measurements. The posterior distribution for each combination of HMF \& SPS model is shown in full in Fig. 7 and 8 .

First, in Figure 3, we show the UVLF predictions subjected to cosmological and HMF uncertainties. At $z \sim 6$, the differences between Sheth et al. (2001) and Tinker 


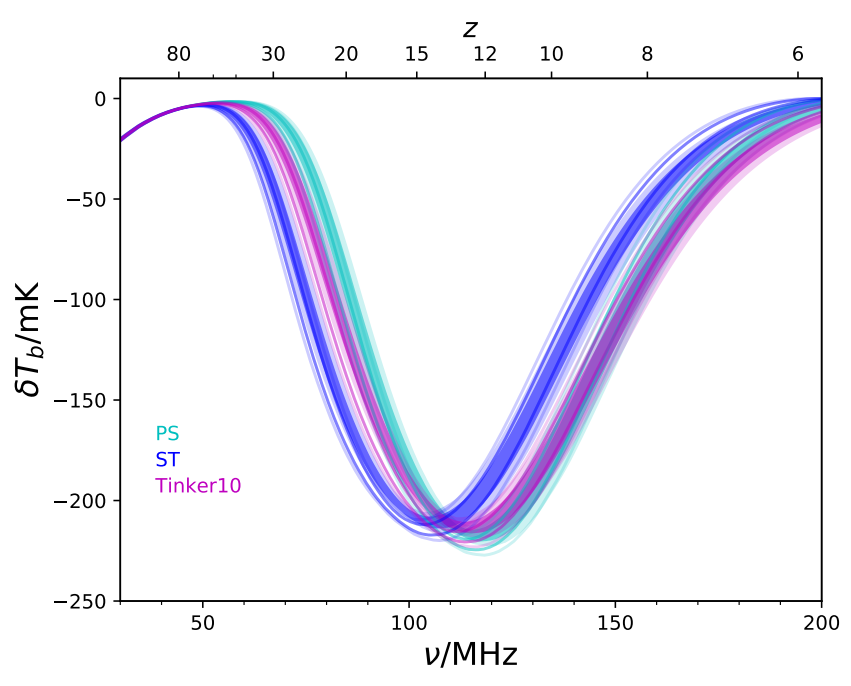

Figure 4. Global 21-cm signal predictions subject to cosmological and HMF uncertainties. The width of each band is set by uncertainties in the cosmological parameters, while each colour indicates a different HMF fitting function. Open contours use BPASs models, while filled contours use starburst99.

et al. (2010) mass functions is comparable to the difference between UVLF measurements reported in Bouwens et al. (2015b) and Finkelstein et al. (2015), though we caution the reader that these two sets of UVLF measurements are obtained via different means and, e.g., make slightly different assumptions about the wavelength corresponding to $M_{\mathrm{UV}}$.

In Figure 4, we show predictions for the global 21-cm signal subject to variations in the cosmological parameters and HMF fitting functions. Qualitatively, the curves are very similar - all exhibiting a deep absorption trough at frequencies of $\nu \simeq 110 \mathrm{MHz}$, in line with the predictions of Mirocha et al. (2017). However, the level of variation is not insignificant, as we show more quantiatively in Figure 5. Here, we show only the position of the absorption minimum $\left(\nu_{\min }\right.$, $\left.\delta T_{b, \min }\right)$, and the Thomson scattering optical depth, $\tau_{e}$. The position in frequency varies by $\sim 10-15 \mathrm{MHz}$ between the different fitting functions, while cosmological uncertainties constitute $\sim 2-3 \mathrm{MHz}$ of these differences for each HMF. Similarly, the amplitude of the absorption trough is uncertain at the level of $\sim 10-15 \mathrm{mK}$, about $\sim 5 \mathrm{mK}$ of which is due solely to uncertainties in cosmological parameters. Finally, $\tau_{e}$ uncertainties are $\sim 0.01$.

In Figure 6, we show the full reionization history for each model. Once again, differences are apparent by eye. For example, at the midpoint of reionization, uncertainties in the cosmological parameters contribute an uncertainty of $\sim 5 \%$ in the mean neutral fraction, while the position of the reionization midpoint itself varies by $\Delta z_{\mathrm{rei}} \simeq 0.5$ from Sheth et al. (2001) to Tinker et al. (2010) mass functions. At first glance, the latter seems to produce a reionization history incompatible with current constraints. However, we emphasize that the escape fraction is held fixed in all of these models. Increasing $f_{\text {esc }}$ from 0.2 to $\sim 0.25-0.3$ is enough to bring the Tinker et al. (2010) models into agreement with the Press \& Schechter (1974) and Sheth et al. (2001) models. Note that our neglect of dust for this exercise is in part responsible
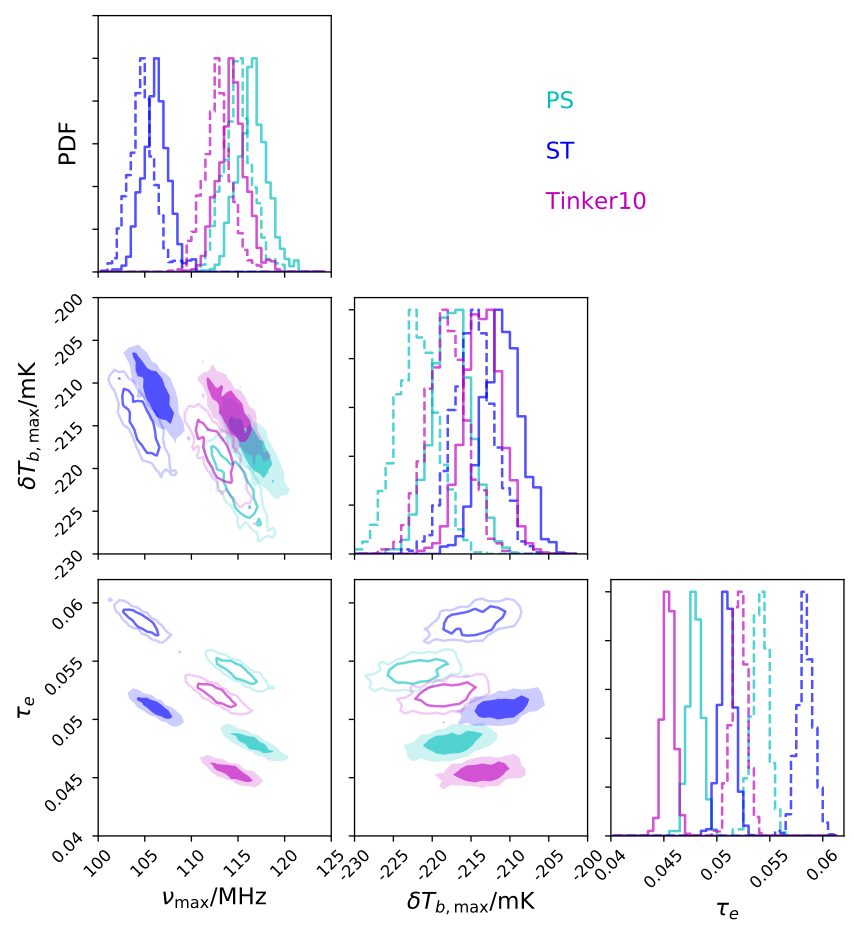

Figure 5. Posterior PDFs of the position of the global 21-cm absorption trough ( $\left.\nu_{\min }, \delta T_{b, \min }\right)$ and Thomson optical depth. Different HMF fitting functions are indicated by colour, while open (filled) contours indicate the use of BPASS (starburst 99 ). The width of the $68 \%$ and $95 \%$ confidence intervals for each individual HMF/SPS pairing are set by uncertainties in the cosmological parameters.

for needing such high escape fractions, since dustier galaxies require more star formation to preserve agreement with UVLFs. We include dust following standard recipes (see §2) in all that follows.

We now turn our attention to the effect these uncertainties have on our ability to constrain the main parameters of our model. To proceed, we create a global 21-cm signal mock generated assuming the Sheth et al. (2001) mass function, BPASS SPS models, and the best-fit Planck cosmology, with $f_{*}$ parameters calibrated to the Bouwens et al. (2015b) UVLFs assuming the Meurer et al. (1999) IRX- $\beta$ and Bouwens et al. (2014) $M_{\mathrm{UV}}-\beta$ relations to correct for dust. We then add spectrally-flat Gaussian random noise with standard deviation $\sigma=5 \mathrm{mK}$ to the global 21-cm mock, which is comparable to the level of uncertainty caused by cosmological parameter variations. We adopt the Bouwens et al. (2015b) UVLFs throughout as our fiducial set of observations to be fit, though similar conclusions would be drawn if we instead adopted the Finkelstein et al. (2015) measurements. We do not include the $z \sim 10$ UVLFs of Oesch et al. (2018) in the analysis.

There is no guarantee that the global $21-\mathrm{cm}$ signal and, e.g., the $z \sim 6-8$ UVLFs from Bouwens et al. (2015a) can both be well-fit simultaneously if we adopt an HMF or SPS model different from that assumed when generating the 21-cm mock. In order to accommodate this possibility, we employ a simple extension to the standard double power-law $f_{*}$ model, multiplying Eq. (2) by a modulation factor (as in 


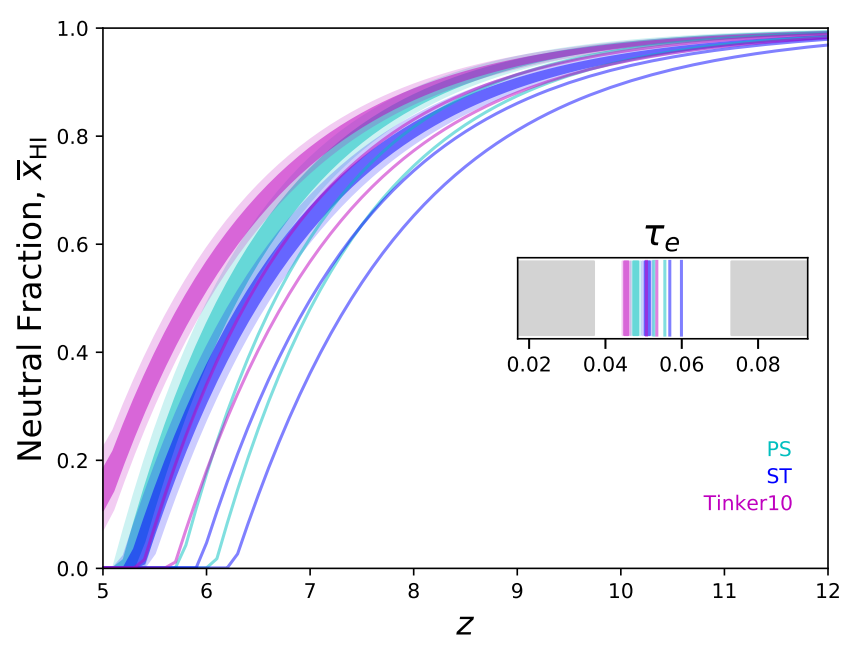

Figure 6. Mean reionization history predictions subject to cosmological and HMF uncertainties. Conventions are the same as Fig. 4. The inset shows the Thomson scattering optical depth shown for each model, with gray regions indicating the range of values excluded at $2 \sigma$ according to the Planck constraint $\tau_{e}=0.055 \pm 0.009$.

Schneider et al., 2020), i.e.,

$$
f_{*} \rightarrow f_{*}\left[1+\left(\frac{M_{h}}{a}\right)^{b}\right]^{c}
$$

which allows $f_{*}$ to depart from a power-law at the low-mass end. This parameterization is appealing because it can capture two qualitatively different possibilities at the low-mass end of the halo population. Depending on the values of $b$ and $c$, the efficiency of star formation can either (i) rapidly decline in halos below $M_{h} \simeq a$, and thus mimic reionization feedback or other processes which inhibit star formation preferentially in low-mass halos, or (ii) boost $f_{*}$ to levels above those predicted by an extrapolation of UVLFs, which could indicate, e.g., very efficient Pop III star formation and/or a failure of stellar feedback within otherwise normal galaxies.

We further allow $f_{*, 10}$ to evolve with redshift as a power-law with index $\gamma_{z}$, resulting in a total of four new parameters. In this work, we treat these as nuisance parameters, though of course departures from power-law and/or redshift-independent $f_{*}$ likely correspond to very interesting scenarios worth constraining. Finally, we allow the four usual SFE parameters to vary freely, as well as the normalization of the X-ray luminosity-SFR relation $\left(L_{X} / \mathrm{SFR}\right)$, escape fraction of ionizing radiation $\left(f_{\text {esc }}\right)$, and minimum virial temperature of star-forming halos, $T_{\min }$. These last three parameters are only constrained by the global 21-cm signal in our framework, as we do not include measurements of the reionization history in our likelihood.

The results of this exercise are shown in Figures 7 and 8. First, in each panel of Fig. 7, we see six posterior distributions: one for each combination of HMF (colours) and SPS model (open vs. filled contours). It is immediately clear that the differences from posterior to posterior are generally larger than the width of any individual posterior distribution. In other words, these systematic modeling uncer- tainties are larger than the uncertainty caused by the error budget of each individual fit $(5 \mathrm{mK}$ Gaussian random noise in global 21-cm signal and errors in UVLF reported by Bouwens et al. 2015b) as well as uncertainty due to marginalizing over cosmological parameters. For reference, we show also the $95 \%$ contours obtained under the assumption of $20 \mathrm{mK}$ level noise in the global 21-cm signal as dotted contours in each interior panel of Fig. 7. In some dimensions, the dotted contours enclose a subset of the individual PDFs, indicating that systematic modeling uncertainties are comparable to those caused by a $20 \mathrm{mK}$ noise level. However, generally speaking, the biases causd by modeling systematics are not mimicked well by inflated uncertainties in the global 21-cm signal.

Though Figure 7 paints a somewhat grim picture in which many parameters are constrained much more poorly than idealized forecasts would suggest, a few panels give reasons to be optimistic. For example, the low-mass slope of the SFE (third column), $\alpha_{\text {lo }}$, while affected by systematics in a relative sense differs little from case to case in an absolute sense - the overall spread is only $\sim 0.1$. For reference, the differences in $\alpha_{\text {lo }}$ for different stellar feedback scenarios is 1/3 (see, e.g., Dayal et al., 2013; Furlanetto et al., 2017), while purely empirical models for the SFE vary by $\delta \alpha_{\text {lo }} \sim$ 0.5 depending on assumptions about, e.g., dust (Mirocha et al., 2017; Tacchella et al., 2018; Mirocha et al., 2020). As a result, it seems that the part of the SFE most easily related to physical arguments is imminently constrainable.

Similarly, the $L_{X}$-SFR normalization (sixth column) is also quite impervious to the systematic uncertainties we explored here - though bimodal with respect to SPS model, the difference is order $\sim 0.1$ dex, which is hardly a cause for concern in differentiating models. More worrisome is the minimum virial temperature, $T_{\mathrm{min}}$, which varies by an order of magnitude from end to end, and the high-mass behavior of the SFE (set by $M_{\text {peak }}$ and $\alpha_{\text {hi }}$ ). However, if we can confidently discard the Press \& Schechter (1974) mass function (cyan contours), the range of viable possibilities in each slice through parameter space is substantially reduced.

Figure 8 focuses on the 'nuisance' parameters introduced to alleviate tension caused by modeling systematics. In reality, these parameters are of interest, as they quantify the degree to which the efficiency of star formation departs from a double power-law in mass and whether it may evolve with redshift. Both effects are expected to some degree, as stellar feedback models predict redshift evolution in $f_{*}$, while reionization feedback and/or efficient Pop III star formation are likely to cause a departure from the double power-law form at low-mass.

\section{DISCUSSION \& CONCLUSIONS}

In this work, we investigated three potential sources of systematic uncertainty in models for high- $z$ galaxies and the mean ionization and thermal history of the IGM.

First, by drawing samples from the Planck MCMC chains, we showed that cosmological uncertainties alone result in $\sim 5 \%$ errors on the mean ionized fraction of the IGM. The global 21-cm signal-which encodes the IGM thermal and ionization histories - is affected by cosmological uncertainties at the level of $\sim 5 \mathrm{mK}$ in the amplitude of its ab- 


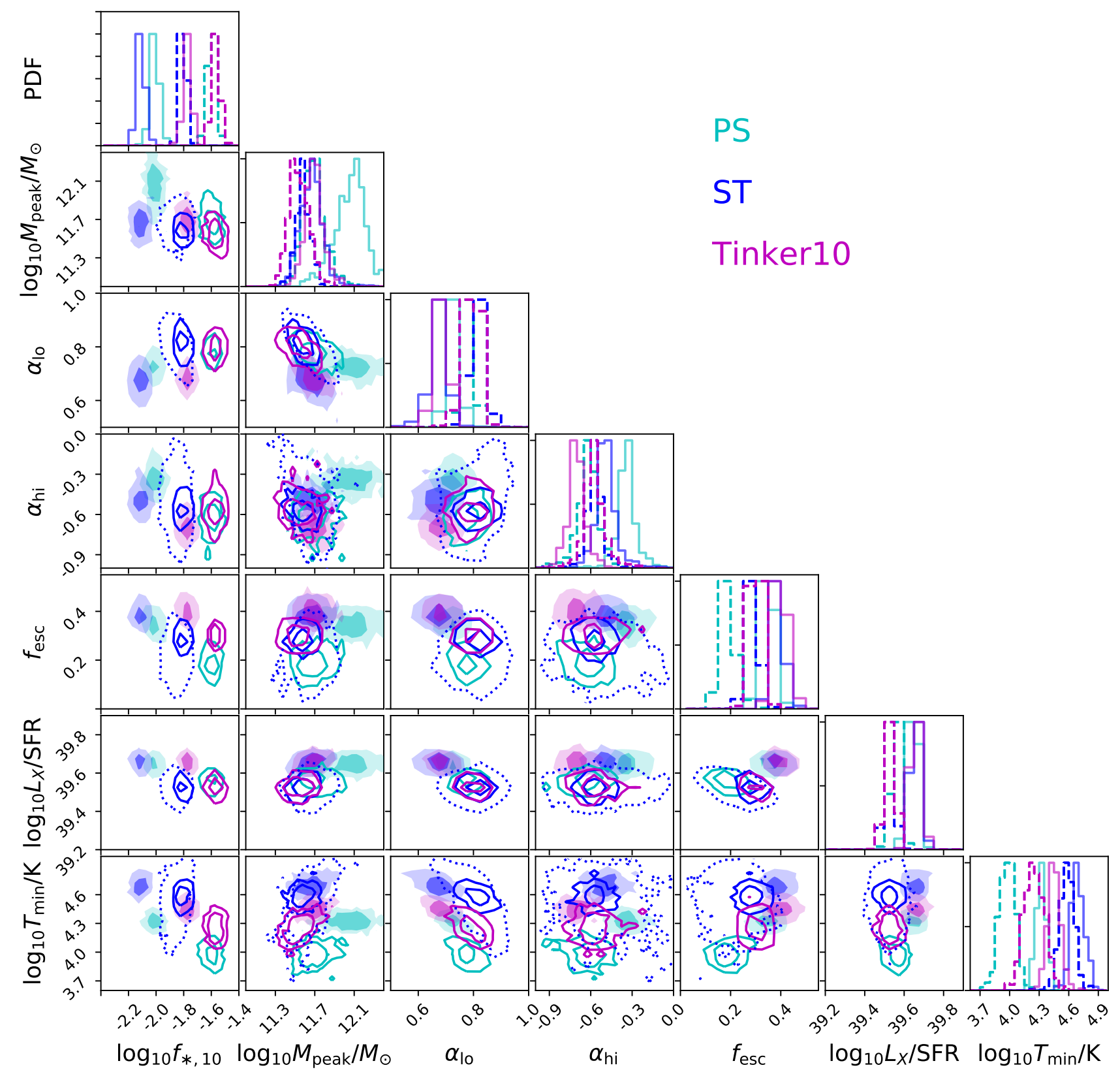

Figure 7. Posterior distributions for six different fits to the same mock global 21-cm signal with $1 \mathrm{mK}$ Gaussian noise and the $z \sim 6$ UVLF from Bouwens et al. (2015b). Colours indicate the different HMFs, while filled (open) contours use of STARBurst99 (BPASS) SPS models. Marginalized 1-D distributions lie along the diagonal, where dashed lines correspond to the open contours of the interior panels. Dotted contours in the interior panels indicate $95 \%$ contours obtained when assuming $20 \mathrm{mK}$, rather than $5 \mathrm{mK}$, Gaussian random noise in the global 21-cm signal mock.

sorption trough, with $\sim 2-3 \mathrm{MHz}$ uncertainties in position. These cosmological uncertainties are the smallest of the three effects we explored.

Next, we adopted three commonly-used halo mass function models, Press \& Schechter (1974), Sheth et al. (2001), and Tinker et al. (2010), as well as two commonly-used stellar population synthesis models, STARBURST99 (Leitherer et al., 1999) and BPASs (Eldridge \& Stanway, 2009), resulting in a total of 6 possible pairings. Differences due to one's choice of HMF and SPS model are larger than those caused by cosmological uncertainties by virtually any metric. For example, variations in the Thomson scattering optical depth, $\tau_{e}$, fill roughly half of the $1 \sigma$ error bar reported by Planck, $\tau_{e}=0.055 \pm 0.009$ (Fig. 6). The strongest feature of the global signal shifts by up to $\sim 15 \mathrm{MHz}$ in frequency and $\sim 20 \mathrm{mK}$ in amplitude (Fig. 4).

To explore how these uncertainties propagate to constraints on model parameters, we performed a joint MCMC fit of a mock global 21-cm signal with currenty high- $z$ UVLF constraints. Global 21-cm forecasts based on thermal noise 
Cosmic dawn modeling systematics

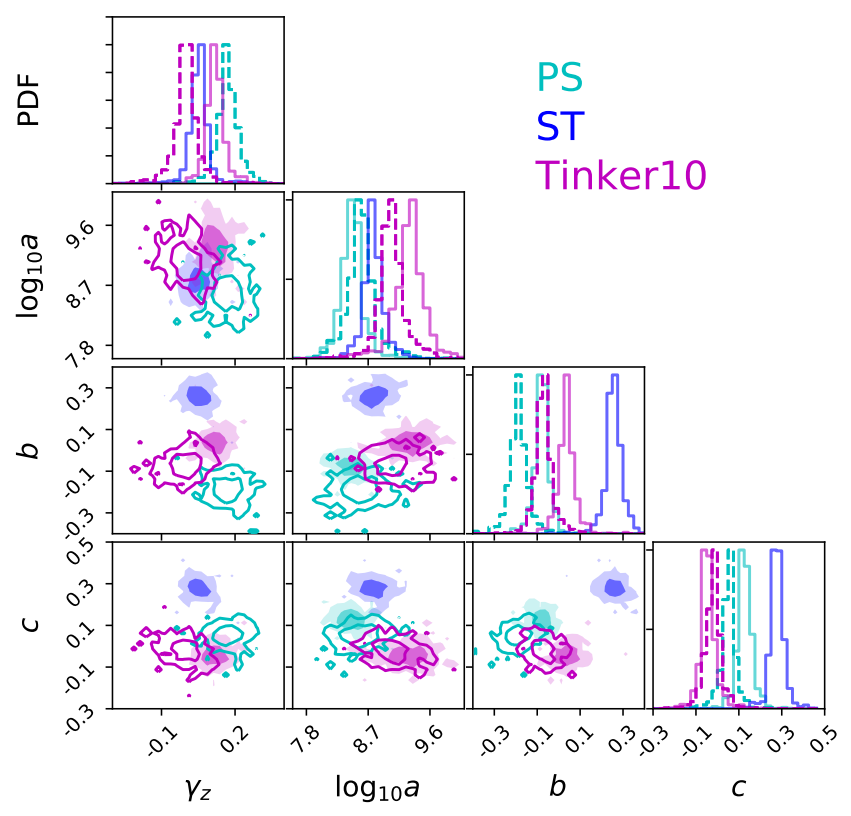

Figure 8. Posterior distributions for four nuisance parameters introduced to relieve tension caused by modeling systematics. For the mock global 21 -cm signal being fit, $f_{*}$ does not evolve with redshift $\left(\gamma_{z}=0\right)$ and the parameters $a, b$, and $c$ (see Eq. 9) are unused.

generally predict tight, $\lesssim 10 \%$ level constraints on model parameters of interest (see, e.g., Mirocha et al., 2015); however, in many dimensions of parameter space, we find that systematic modeling uncertainties result in factor of $\sim 2$ to 3 uncertainties in best-fit parameter values. Fortunately, two highly sought-after parameters - $\alpha_{\text {lo }}$ and $L_{X} / \mathrm{SFR}$ - are relatively impervious to systematic uncertainties, leaving the expectations from idealized forecasts largely unchanged.

There are many as-yet-unexplored sources of systematic modeling uncertainty in the reionization context. For example, we have completely neglected any numerical shortcomings of our model, e.g., the accuracy of the two-zone model of the IGM, correlations between the fields composing the 21$\mathrm{cm}$ background, or the idealized approach to high- $z$ galaxy evolution. Of course, more detailed 3-dimensional reionization calculations sourced with galaxies modeled via semianalytic prescriptions are likely to be more realistic in such regards. However, more sophisticated models have systematic uncertainties as well, caused by, e.g., approximate radiative transfer techniques and sub-grid models for sources and sinks. Similarly, extending models to redshifts $z \ll 6$ can in principle tighten constraints on parameters of interest, but may also introduce yet more systematic uncertainties (Behroozi et al., 2010).

Given the ever-improving constraints on reionization, it is important to continue efforts to quantify the theoretical modeling error budget. Doing so will help to properly report uncertainties on parameters of interest, and identify the biggest problem areas and thus guide dedicated efforts to mitigate them. For some applications, eliminating systematics may be unnecessary, since some parameters of interest cease to be meaningful at arbitrarily high levels of precision. For example, most parameters in 21-cm mod- els are averages over entire galaxy populations, with reference points anchored to uncertain empirical scaling relationships $\left(L_{X} / \mathrm{SFR}\right)$ for which even constraints at the factor of $\sim 2-3$ level are interesting. However, tests of the cosmological model, e.g., non-cold dark matter, is an intrinsically more quantitative enterprise, in which all sources of systematic uncertainty must be understood or eliminated. In these contexts, there is much work still to be done before limits (or constraints) on various exotic models can be trusted to high precision.

It may be possible to avoid detailed characterization of modeling uncertainties and instead use model selection techniques (e.g., nested sampling; Skilling, 2006) to constrain modeling assumptions from empirical measurements themselves. There are now several publicly-available tools for model selection (e.g., Feroz \& Hobson, 2008; Handley et al., 2015), which are becoming more frequently applied in 21cm contexts (e.g., Binnie \& Pritchard, 2019; Sims \& Pober, 2020). While the ultimate goal is to differentiate competing models of galaxy formation and reionization, model selection tools can of course also be used to discern the presence of systematics, including those associated with instruments (Sims \& Pober, 2020), but in principle also systematics associated with signal modeling. The addition of information from 21-cm interferometers would surely help this effort as spatial fluctuations in the 21-cm background are of course also sensitive to the HMF (see, e.g., Lopez-Honorez et al., 2016), but also encode the bias of UV and X-ray sources, and should thus mitigate the many degeneracies that impede inference based solely on the mean reionization and thermal histories, like those we focus on here. We defer such analyses to future work.

In this paper we have conducted a first study of how uncertainties in theoretical models can affect interpretations of current and upcoming probes of cosmic dawn. Although the conclusions may seem pessimistic at first glance, they in fact demonstrate the excitement that will accompany the expected observational progress of the next few years: rather than a scenario where experiments will merely fine tune a handful of model parameters, we will be in a situation where there exists a truly symbiotic relationship between theory and experiment, with each side informing the other in future improvements. Together, they will lead the community toward a new understanding of our cosmic dawn.

\section{ACKNOWLEDGMENTS}

The authors thank Saurabh Singh, Brad Greig, Peter Sims, and Elizabeth Stanway for helpful conversations that improved this manuscript, and the anonymous referee for many useful suggestions that helped clarify the text. J.M. acknowledges support from a CITA National Fellowship. H.L. was supported by a Science Undergraduate Research Award from the McGill University Faculty of Science. A.L. acknowledges support from the New Frontiers in Research Fund Exploration grant program, a Natural Sciences and Engineering Research Council of Canada (NSERC) Discovery Grant and a Discovery Launch Supplement, a Fonds de recherche Nature et echnologies Quebec New Academics grant, the Sloan Research Fellowship, the William Dawson Scholarship at McGill, as well as the Canadian Institute for Advanced Re- 
search (CIFAR) Azrieli Global Scholars program. Computations were made on the supercomputer Cedar at Simon Fraser University managed by Compute Canada. The operation of this supercomputer is funded by the Canada Foundation for Innovation (CFI).

Software: numpy (Van Der Walt et al., 2011), scipy (Virtanen et al., 2020), matplotlib (Hunter, 2007), h5py ${ }^{3}$, and mpi4py (Dalcín et al., 2005).

Data Availability: The data underlying this article is available upon request, but can also be re-generated from scratch using the publicly available ARES code.

\section{References}

Barkana R., 2018, Nat, 555, 71

Barkana R., Loeb A., 2005, ApJ, 626, 1

Behroozi P. et al., 2020, MNRAS, 499, 5702

Behroozi P. et al., 2019, MNRAS, 488, 3143

Behroozi P. S., Conroy C., Wechsler R. H., 2010, ApJ, 717, 379

Bhattacharya S. et al., 2011, ApJ, 732, 122

Binnie T., Pritchard J. R., 2019, MNRAS, 487, 1160

Boddy K. K. et al., 2018, Phys Rev D, 98, 123506

Bouwens R. J. et al., 2015a, ApJ, 811, 140

Bouwens R. J. et al., 2014, ApJ, 793, 115

Bouwens R. J. et al., 2015b, ApJ, 803, 34

Bowman J. D. et al., 2018, Nat, 555, 67

Chluba J., Thomas R. M., 2011, MNRAS, 412, 748

Cohen A. et al., 2020, MNRAS, 495, 4845

Dalcín L., Paz R., Storti M., 2005, Journal of Parallel and

Distributed Computing, 65, 1108

Dayal P. et al., 2013, MNRAS, 434, 1486

Eldridge J. J., Stanway E. R., 2009, MNRAS, 400, 1019

Eldridge J. J. et al., 2017, PASA, 34, e058

Feroz F., Hobson M. P., 2008, MNRAS, 384, 449

Fialkov A., Barkana R., Cohen A., 2018, PRL, 121, 011101

Fialkov A. et al., 2013, MNRAS, 432, 2909

Field G. B., 1958, Proceedings of the IRE, 46, 240

Finkelstein S. L. et al., 2019, ApJ, 879, 36

Finkelstein S. L. et al., 2015, ApJ, 810, 71

Foreman-Mackey D. et al., 2013, PASP, 125, 306

Furlanetto S. R., 2006, MNRAS, 371, 867

Furlanetto S. R. et al., 2017, MNRAS, 472, 1576

Greig B., Mesinger A., 2015, MNRAS, 449, 4246

Greig B., Mesinger A., 2017, MNRAS, 472, 2651

Haardt F., Madau P., 1996, ApJ, 461, 20

Handley W. J., Hobson M. P., Lasenby A. N., 2015, Monthly Notices of the Royal Astronomical Society: Letters, 450, L61

Hunter J. D., 2007, Computing in Science \& Engineering, 9,90

Hutter A., 2018, MNRAS, 477, 1549

Hutter A. et al., 2020, arXiv e-prints, arXiv:2004.08401

Kern N. S. et al., 2017, ApJ, 848, 23

Knebe A. et al., 2013a, MNRAS, 428, 2039

Knebe A. et al., 2013b, MNRAS, 435, 1618

Leitherer C. et al., 1999, ApJS, 123, 3

Lewis A., Challinor A., Lasenby A., 2000, ApJ, 538, 473

3 http://www.h5py.org/
Liu A. et al., 2016, Phys Rev D, 93, 043013

Liu A., Shaw J. R., 2020, PASP, 132, 062001

Lopez-Honorez L. et al., 2016, JCAP, 8, 004

Majumdar S. et al., 2014, MNRAS, 443, 2843

Mason C. A., Trenti M., Treu T., 2015, ApJ, 813, 21

Mesinger A., Ferrara A., Spiegel D. S., 2013, MNRAS, 431, 621

Mesinger A., Furlanetto S., 2007, ApJ, 669, 663

Mesinger A., Furlanetto S., Cen R., 2011, MNRAS

Meurer G. R., Heckman T. M., Calzetti D., 1999, ApJ, 521, 64

Mirocha J., 2014, MNRAS, 443, 1211

Mirocha J., 2020, MNRAS, 499, 4534

Mirocha J., Furlanetto S. R., Sun G., 2017, MNRAS, 464, 1365

Mirocha J., Harker G. J. A., Burns J. O., 2015, ApJ, 813, 11

Mirocha J., Mason C., Stark D. P., 2020, MNRAS, 498, 2645

Mitsuda K. et al., 1984, PASJ, 36, 741

Monsalve R. A. et al., 2017, ApJ, 847, 64

Muñoz J. B., Cyr-Racine F.-Y., 2020, arXiv e-prints, arXiv:2005.03664

Muñoz J. B., Dvorkin C., Cyr-Racine F.-Y., 2020, Phys Rev D, 101, 063526

Muñoz J. B., Loeb A., 2018, Nat, 557, 684

Murray S. G., Power C., Robotham A. S. G., 2013a, Astronomy and Computing, 3, 23

Murray S. G., Power C., Robotham A. S. G., 2013b, $M N$ $R A S, 434, \mathrm{~L} 61$

Oesch P. A. et al., 2018, ApJ, 855, 105

Park J. et al., 2019, MNRAS, 484, 933

Planck Collaboration et al., 2018, arXiv e-prints, arXiv:1807.06209

Press W. H., Schechter P., 1974, ApJ, 187, 425

Pritchard J. R., Furlanetto S. R., 2007, MNRAS, 376, 1680

Pritchard J. R., Loeb A., 2010, Physical Review D, 82, 23006

Pritchard J. R., Loeb A., 2012, Reports on Progress in Physics, 75, 086901

Qin Y. et al., 2020, MNRAS, 495, 123

Reed D. S. et al., 2007, MNRAS, 374, 2

Reichardt C. L. et al., 2020, arXiv e-prints, arXiv:2002.06197

Robertson B. E. et al., 2015, ApJL, 802, L19

Santos M. G. et al., 2010, MNRAS, 406, 2421

Schneider A., 2018, Phys Rev D, 98, 063021

Schneider A., Giri S., Mirocha J., 2020, arXiv e-prints, arXiv:2011.12308

Sheth R. K., Mo H. J., Tormen G., 2001, MNRAS, 323, 1

Sims P. H., Pober J. C., 2020, MNRAS, 492, 22

Singh S. et al., 2018, ApJ, 858, 54

Sitwell M. et al., 2014, MNRAS, 438, 2664

Skilling J., 2006, Bayesian Analysis, 1, 833

Sun G., Furlanetto S. R., 2016, MNRAS, 460, 417

Tacchella S. et al., 2018, ApJ, 868, 92

Tashiro H., Kadota K., Silk J., 2014, Phys Rev D, 90, 083522

Tinker J. L. et al., 2010, ApJ, 724, 878

Trac H., Cen R., Mansfield P., 2015, ApJ, 813, 54

Van Der Walt S., Colbert S. C., Varoquaux G., 2011, Computing in Science \& Engineering, 13, 22 
Virtanen P. et al., 2020, Nature Methods, 17, 261

Warren M. S. et al., 2006, ApJ, 646, 881

Williams C. C. et al., 2018, The Astrophysical Journal Supplement Series, 236, 33

Wouthuysen S. A., 1952, AJ, 57, 31

Yung L. Y. A. et al., 2019, MNRAS, 483, 2983

Zahn O. et al., 2011, MNRAS, 414, 727

Zygelman B., 2005, ApJ, 622, 1356 